\title{
Complicaciones tempranas en los primeros 50 casos de cirugía mínimamente invasiva lateral a la columna lumbar
}

\author{
Juan J. Gottlieb, Horacio Resano \\ Sección Patología de Columna Vertebral, Hospital Sirio Libanés, ECICARO, Ciudad Autónoma de Buenos Aires, Argentina
}

\begin{abstract}
RESUMEN
Introducción: Las técnicas mínimamente invasivas intentan mejorar la morbilidad posoperatoria en las fusiones lumbares. Esta limitación en el abordaje quirúrgico y la potencial falta de visión del campo operatorio no estarían exentas de complicaciones. El objetivo de este estudio fue analizar nuestra tasa de complicaciones tempranas posoperatorias, en pacientes sometidos a un abordaje mínimamente invasivo lateral, valorando las complicaciones en relación con nuestra curva de aprendizaje. Materiales y Métodos: Estudio retrospectivo de 50 pacientes operados con técnica de artrodesis intersomática por vía lateral retroperitoneal mínimamente invasiva, entre septiembre de 2015 y abril de 2018. Los parámetros incluidos fueron: demográficos, radiológicos, quirúrgicos y todas las complicaciones tempranas. Resultados: Se colocaron 76 cajas: 5 en L1-L2, 33 en L2-L3, 34 en L3-L4 y 4 en L4-L5. A 25 de los 50 pacientes se les realizó un solo nivel de fusión; a 24, dos niveles y a uno, tres niveles. Se detectaron 21 complicaciones (4 mayores y 17 menores). El tiempo quirúrgico más prolongado (90 min) y la tasa más alta de complicaciones correspondieron a los casos 1-16 (12 pacientes), luego estas cifras disminuyeron proporcionalmente en los siguientes casos hasta llegar a un mínimo de tres complicaciones en los casos 34-50. Conclusiones: Las mayores complicaciones ocurrieron en la vía de abordaje, pero disminuyeron progresivamente, a medida que crecía la experiencia del cirujano y se acortaba el tiempo quirúrgico. Palabras clave: Abordaje lateral lumbar; técnica XLIF; complicaciones.
\end{abstract}

Nivel de Evidencia: IV

Early complications of the minimally invasive lateral lumbar spine surgery: a 50-case series

\begin{abstract}
Introduction: Minimally invasive spine surgeries (MISS) are designed to improve postoperative morbidity in lumbar fusion procedures. Their limited surgical exposure and potentially poor visualization of the operative field are not without complications. The purpose of our study was to analyze our early postoperative complication rate in the first 50 patients that underwent a lateral MISS approach, evaluating these procedures in relation to our learning curve. Materials and Methods: We conducted a retrospective study in 50 patients that underwent lateral retroperitoneal interbody fusion using a MISS technique between September 2015 and April 2018. The assessed parameters were: demographic, radiological and surgical data, and all the early complications encountered. Results: A total of 76 cages were used: 5 at L1-L2 level; 33 at L2-L3 level; 34 at L3-L4 level; and 4 at L4-L5 level. Out of the 50 patients, 25 underwent fusion only at 1 level, 24 underwent fusion at 2 levels, and 1 underwent fusion at 3 levels. A total of 21 complications were encountered: 4 major complications, and 17 minor complications. The longest surgical time (90 minutes) and the highest complication rate were observed within cases 1-16 (12 patients). Thereafter, these parameters proportionately decreased until they reached a minimum of 3 complications within cases 34-50. Conclusions: The main complications occurred in the surgical access, and their incidence decreased as the surgeon's experience increased and as the surgical time shortened. Key words: Lumbar lateral approach; XLIF; complications.
\end{abstract}

Level of Evidence: IV

Recibido el 22-3-2019. Aceptado luego de la evaluación el 12-8-2019 • Dr. JUAN J. GOTTLIEB • juangottlieb@ hotmail.com (ID

Cómo citar este artículo: Gottlieb JJ, Resano H. Complicaciones tempranas en los primeros 50 casos de cirugía mínimamente invasiva lateral a la columna lumbar. Rev Asoc Argent Ortop Traumatol 2020;85(1):39-48. https://doi.org/10.15417/issn.1852-7434.2020.85.1.970 


\section{INTRODUCCIÓN}

Se ha descrito a la fusión lumbar como una exitosa técnica quirúrgica para el tratamiento de múltiples patologías espinales (discopatías degenerativas, trauma, tumores, etc.) y como la indicación estándar para varias de ellas.

La fusión posterolateral a cielo abierto sigue siendo el tratamiento de elección en la cirugía de columna, pero la gran pérdida de sangre, el dolor lumbar posoperatorio y cierta dificultad quirúrgica motivaron el desarrollo de nuevos métodos para la fijación intersomática, que disminuyeran la disrupción de la musculatura espinal en los abordajes y, por ende, el dolor y la morbilidad posoperatorios.

Se han comunicado numerosas técnicas mínimamente invasivas con el objetivo de limitar la exposición quirúrgica, disminuir la pérdida de sangre y la estancia hospitalaria, y mejorar la morbilidad posoperatoria. Esta limitación en el abordaje quirúrgico y la potencial falta de visión del campo operatorio no estarían exentas de complicaciones.

Unas de estas técnicas mínimamente invasivas que se ha popularizado en los últimos años es la fusión intersomática lateral retroperitoneal por vía transpsoas, desarrollada por el doctor Pimenta, ${ }^{1}$ de Brasil, y publicada en 2006. Este abordaje lateral permite realizar una discectomía dejando intactos los ligamentos vertebral común anterior y posterior, proporcionando más estabilidad y un mayor índice de contacto caja-hueso que favorece la fusión. Otro beneficio del método es realizar correcciones secuenciales coronales y, a veces, sagitales en ciertas deformidades del adulto. Pero así como ofrece grandes ventajas, existirían potenciales riesgos de comprometer estructuras vecinas al cuerpo vertebral.

En la bibliografía internacional, se ha publicado una limitada cantidad de estudios que informen sobre las complicaciones tempranas de esta técnica, pero no hay ningún artículo nacional que las mencione.

El objetivo de este estudio retrospectivo fue analizar nuestra tasa de complicaciones tempranas operatorias, en los primeros 50 pacientes sometidos a un abordaje lateral mínimamente invasivo, valorándolas en relación con la curva de aprendizaje.

\section{MATERIALES Y MÉTODOS}

Se llevó a cabo un estudio retrospectivo basado en datos clínicos y radiográficos de pacientes sometidos a cirugía entre septiembre de 2015 y abril de 2018. La muestra incluyó a 50 pacientes operados, por un mismo cirujano, con la técnica de artrodesis intersomática por vía lateral retroperitoneal mínimamente invasiva.

Los pacientes fueron evaluados por dos miembros del equipo quirúrgico dentro de los 45 días posteriores a la cirugía y la revisión retrospectiva se completó en una lista de datos, compilada por separado. Los parámetros incluidos fueron: demográficos (sexo y edad), radiográficos (cantidad de niveles, niveles fusionados y fijación posterior o no) y quirúrgicos (diagnóstico preoperatorio y sistema utilizado), y también todas las complicaciones que, para evitar cualquier omisión, fueron categorizadas en otra lista confeccionada por el equipo quirúrgico, tomando como referencia, la frecuencia observada en la bibliografía internacional.

Para evaluar la curva de aprendizaje, su relación con los episodios adversos y el tiempo quirúrgico, los casos fueron divididos en tres partes (del caso 1 al 16, del 17 al 33 y del 34 al 50).

La técnica quirúrgica se efectuó según los pasos descritos por el doctor Pimenta, pero con una modificación: una sola incisión lateral, que es la más utilizada actualmente, en el mundo. Esta técnica se llevó a cabo con anestesia general y el paciente en decúbito lateral, con identificación y posicionamiento bajo fluoroscopia, abordaje y disección según técnica transpsoas, discectomía y colocación de una caja de PEEK intersomática, usando el sistema separador para XLIF® (NuVasive®, San Diego, CA, EE.UU.) en 44 casos, el separador para DLIF® (Medtronic Sofamor Danek, Memphis, TN, EE.UU.) en cuatro casos y el LLIF® (Globus Medical Inc. Aubudon, PA, EE.UU.) en dos casos (total 50 casos), todos bajo monitoreo neurofisiológico intraoperatorio directo.

\section{RESULTADOS}

Treinta y siete mujeres y 13 hombres (edad promedio 68 [rango 44-80] y 67 años [rango 45-78], respectivamente) fueron sometidos a un abordaje lateral mínimamente invasivo.

Se colocaron 76 cajas, distribuidas de la siguiente manera: cinco en L1-L2, 33 en L2-L3, 34 en L3-L4 y cuatro en L4-L5.

A 25 de los 50 pacientes se les realizó un solo nivel de fusión (50\%); a 24, dos niveles (48\%) y a uno, tres niveles de fusión $(2 \%)$.

Solo seis casos se dejaron sin instrumentación ("stand alone"), a los 44 restantes se les practicó una suplementación posterior (Tabla 1). 
Tabla 1. Cantidad de cajas colocadas, niveles de fusión y suplementación posterior

\begin{tabular}{|c|c|c|c|c|c|}
\hline Niveles de fusión & 76 cajas & L1-L2 (5) & L2-L3 (33) & L3-L4 (34) & L4-L5 (4) \\
\hline Cantidad de niveles & $\begin{array}{c}25 \text { casos } \\
1 \text { nivel }\end{array}$ & $\begin{array}{l}24 \text { casos } \\
2 \text { niveles }\end{array}$ & $\begin{array}{c}1 \text { caso } \\
3 \text { niveles }\end{array}$ & & \\
\hline Fijación posoperatoria & 6 sin fijar & 36 abierta & 5 percutánea & 3 unilateral & \\
\hline
\end{tabular}

En esta serie, las discopatías degenerativas con estenosis del canal o sin ella fueron la indicación más común, seguida de los síndromes de adición y la escoliosis del adulto (Tabla 2).

Tabla 2. Diagnóstico preoperatorio y sistema de instrumentación utilizado

\begin{tabular}{|l|l|l|l|}
\hline Diagnóstico preoperatorio & Escoliosis (12) & Síndrome de adición (18) & Discopatía degenerativa/canal estrecho (20) \\
\hline Sistema utilizado & NuVasive ${ }^{\circledR}(44)$ & Medtronic (4) & Globus (2) \\
\hline
\end{tabular}

En el primer tercio de los pacientes operados, se observó el tiempo quirúrgico más prolongado (promedio 90 min) y la tasa más alta de complicaciones (12 casos, 24\%), luego bajó proporcionalmente en los siguientes tercios hasta llegar a una tasa del 6\% de complicaciones en los casos 34-50 (Tabla 3). Se detectaron 21 complicaciones (42\%) en los 50 casos: cuatro fueron mayores (8\%) y 17, menores (34\%). Denominamos complicación mayor a los eventos que fueron crónica y funcionalmente limitantes o que requirieron de una cirugía de revisión para solucionarlos y complicación menor, a aquella que no dejó ninguna secuela, ni requirió una cirugía posterior.

Tabla 3. Complicaciones relacionadas con la curva de aprendizaje y el tiempo quirúrgico

\begin{tabular}{|c|c|c|c|}
\hline $\begin{array}{l}\text { Complicaciones relacionadas } \\
\text { con la curva de aprendizaje }\end{array}$ & Cantidad & Descripción & Tiempo quirúrgico \\
\hline Casos del 1 al 16 & 12 & $\begin{array}{l}\text { Sensitivas y dolor ( } 9 \text { casos) } \\
\text { Lesión de la arteria segmentaria ( } 1 \text { caso) } \\
\text { Ruptura del ligamento longitudinal anterior ( } 1 \text { caso) } \\
\text { Seudohernia ( } 1 \text { caso) }\end{array}$ & 90 min promedio \\
\hline Casos del 17 al 33 & 6 & $\begin{array}{l}\text { Migración lateral ( } 1 \text { caso) } \\
\text { Subsidencia ( } 2 \text { casos }) \\
\text { Mala posición de la caja ( } 3 \text { casos })\end{array}$ & 40 min promedio \\
\hline Casos del 34 al 50 & 3 & $\begin{array}{l}\text { Fractura del cuerpo ( } 1 \text { caso) } \\
\text { Subsidencia ( } 2 \text { casos) }\end{array}$ & 25 min promedio \\
\hline
\end{tabular}


Entre los cuatro pacientes que sufrieron una complicación mayor, dos no fueron sometidos a ninguna cirugía de revisión, pero uno de ellos quedó con dolor neuropático crónico intercostal, se trató de un nivel L1-L2 en donde se resecó parte de la costilla para poder acceder al espacio, y el otro caso fue una escoliosis del adulto en la que se realizó un acceso a los espacios L2-L3 y L3-L4 (el ápice de la curva), y el paciente quedó con una disestesia en la cara anterior de muslo, que continuaba al año de la cirugía. El tercer paciente con una complicación mayor tuvo una lesión de la arteria segmentaria en el espacio L3-L4, la cual se pudo ligar, con la ayuda de un cirujano vascular, por la misma incisión y fue necesario realizar una vía posterior, alterando la estrategia quirúrgica original. En el posoperatorio inmediato del cuarto paciente, se observó una fractura longitudinal del cuerpo de L3, se le colocó una caja en L2-L3 que quedó muy anterior y una caja en el nivel inmediato inferior (L3-L4) colocada más posterior, esto generó una debilidad de ambos platillos vertebrales del cuerpo de L3, por donde se produjo el trazo fracturario, que se solucionó mediante una fijación posterior complementaria (Figura 1).
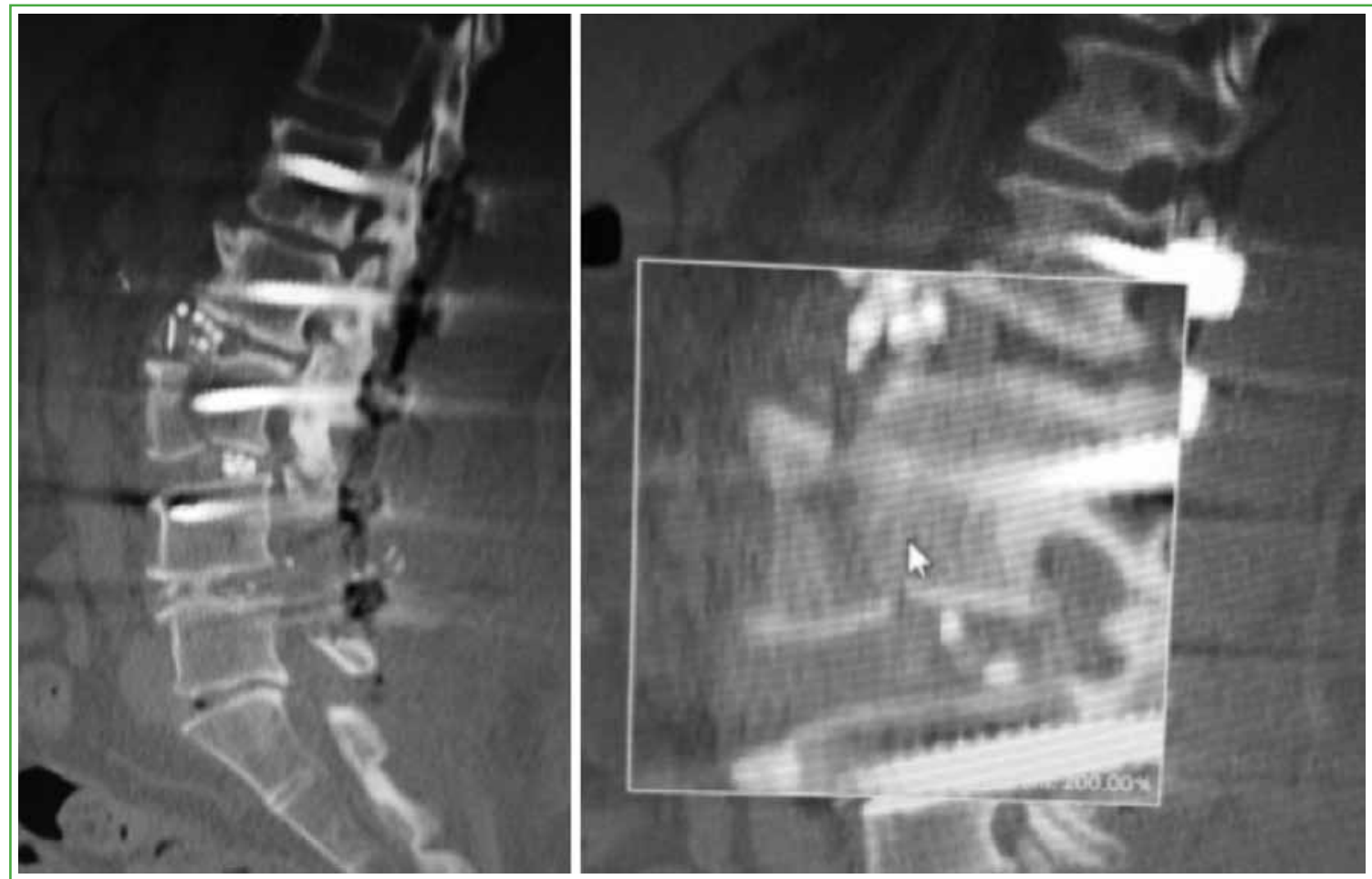

Figura 1. Paciente con fractura del cuerpo de L3.

Los 17 casos restantes fueron complicaciones menores: siete tuvieron alguna alteración sensitiva o debilidad en la flexión de la cadera que se resolvió dentro de las tres semanas posteriores a la cirugía. Un paciente con una paresia de los músculos abdominales (seudohernia) que se resolvió espontáneamente dentro de los tres meses posoperatorios, y otro con una ruptura no intencional del ligamento vertebral común anterior, que fue reparada con una caja atornillada en el mismo momento, quedó un poco anterior al cuerpo vertebral y luego se realizó una instrumentación posterior.

Hubo cuatro casos con subsidencia de la caja intersomática: tres en el platillo inferior, detectada en el primer control, a los 15 días, y uno en el platillo superior, observada en el posoperatorio inmediato (Figura 2). Se detectaron dos casos con migración de la caja (una lateral y otra anterior) en el primer control posoperatorio (Figura 3) y otros dos con mala posición de las cajas, muy laterales, sin secuelas clínicas en los controles sucesivos. 


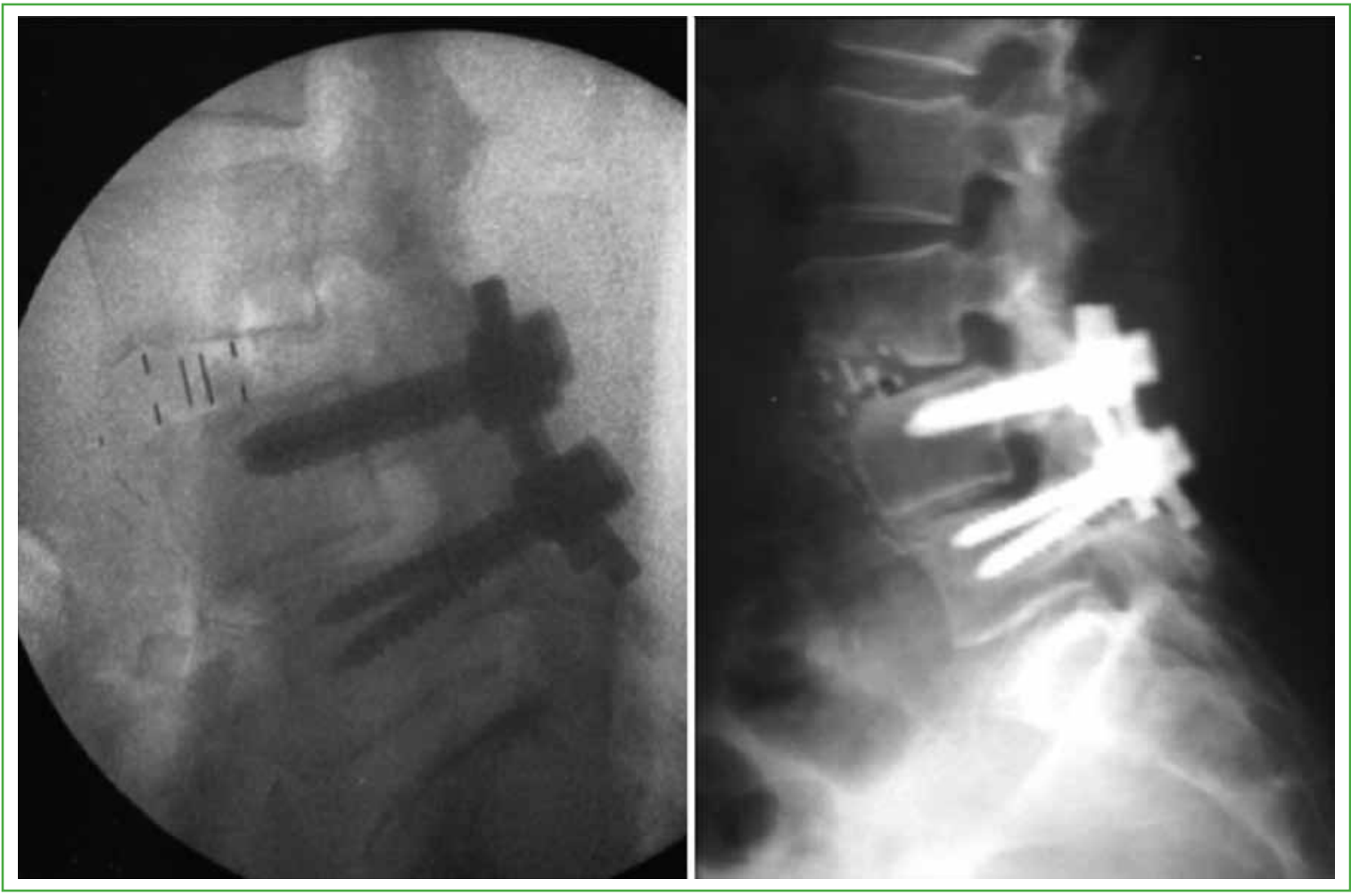

Figura 2. Paciente con subsidencia del platillo superior. Controles intraoperatorio y posoperatorio inmediato.

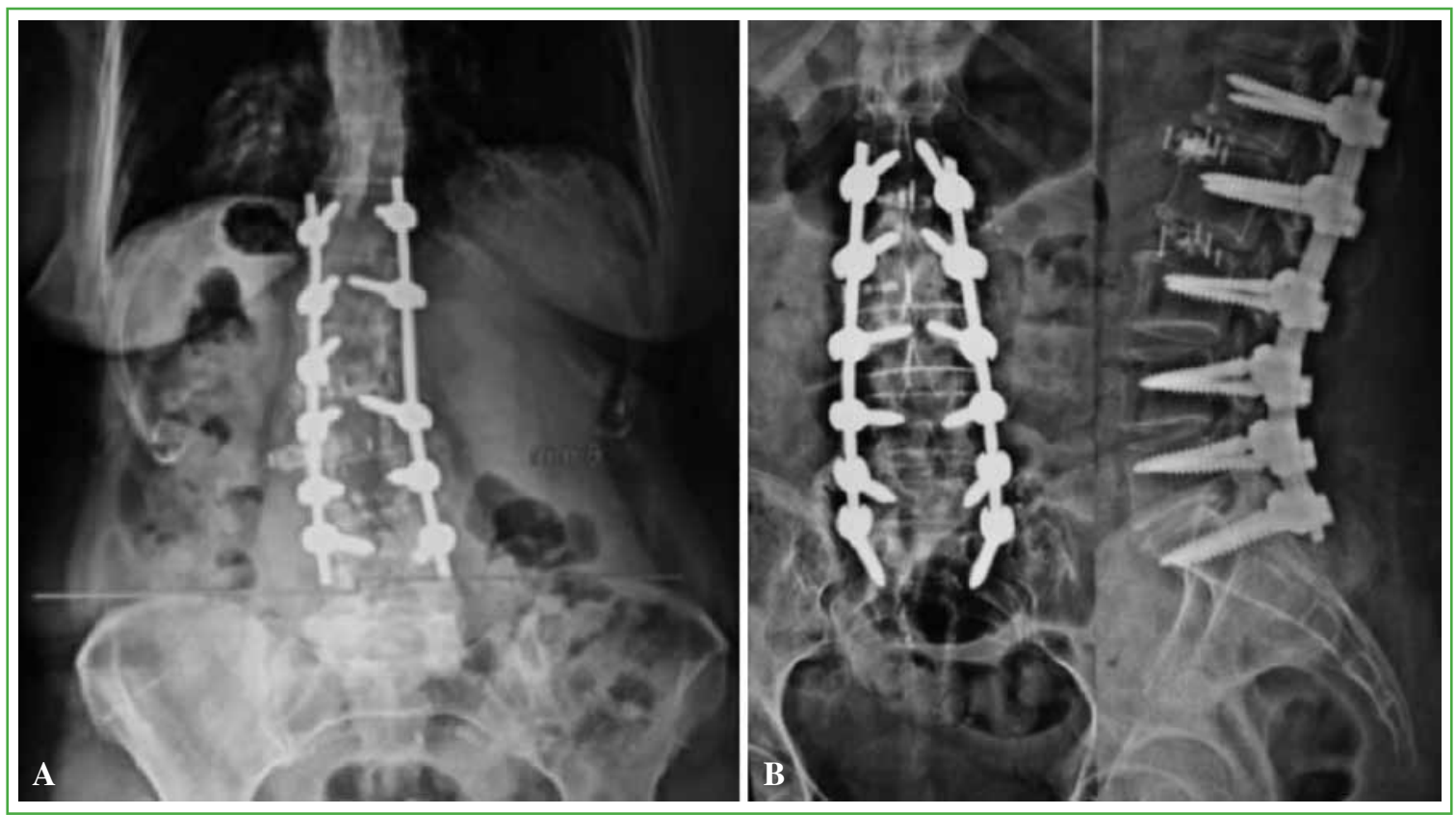

Figura 3. A. Migración lateral. B. Migración anterior. 
No hubo complicaciones motoras ni viscerales, ni infecciones o hematomas retroperitoneales (Tablas 4 y 5).

Tabla 4. Complicaciones relacionadas con el abordaje

\begin{tabular}{|l|c|l|}
\hline $\begin{array}{l}\text { Complicaciones relacionadas } \\
\text { con el abordaje }\end{array}$ & $\begin{array}{c}\text { Cantidad } \\
(\%)\end{array}$ & Descripción \\
\hline Motora & $0(0 \%)$ & \\
\hline Sensitiva & $2(4 \%)$ & $\begin{array}{l}\text { Dolor intercostal crónico (1 caso) } \\
\text { Disestesia crónica de muslo (1 caso) }\end{array}$ \\
\hline Dolor/debilidad del psoas & $7(14 \%)$ & $\begin{array}{l}\text { Dolor a la flexión de la cadera (5 casos) } \\
\text { Debilidad (2 casos) }\end{array}$ \\
\hline Vascular & $1(2 \%)$ & Lesión de la arteria segmentaria vertebral \\
\hline Viscerales & $0(0 \%)$ & \\
\hline Infecciones & $0(0 \%)$ & \\
\hline Hernia/seudohernia abdominal & $1(2 \%)$ & Paresia de músculos abdominales, nivel 2/3 \\
\hline Hematoma retroperitoneal & $0(0 \%)$ & \\
\hline
\end{tabular}

Tabla 5. Complicaciones relacionadas con la técnica quirúrgica

\begin{tabular}{|l|c|l|}
\hline $\begin{array}{l}\text { Complicaciones relacionadas con la técnica } \\
\text { quirúrgica }\end{array}$ & $\begin{array}{c}\text { Cantidad } \\
(\%)\end{array}$ & Descripción \\
\hline Ruptura del ligamento longitudinal anterior & $1(2 \%)$ & Caja en posición anterior atornillada \\
\hline Fractura del cuerpo/subsidencia & $5(10 \%)$ & $\begin{array}{l}\text { Fractura del cuerpo de L3 longitudinal (1 caso) } \\
\text { Subsidencia (4 casos) }\end{array}$ \\
\hline Mal posicionamiento de la caja/migración & $4(8 \%)$ & $\begin{array}{l}\text { Mala posición lateral (2 casos) } \\
\text { Una migración anterior y una lateral (2 casos) }\end{array}$ \\
\hline
\end{tabular}

\section{DISCUSIÓN}

Ningún tratamiento médico-quirúrgico está libre de complicaciones. El avance tecnológico busca disminuir, al mínimo, los posibles efectos adversos durante los procedimientos quirúrgicos y después de la intervención. Las cirugías mínimamente invasivas tienden a buscar estos resultados, tratando de disminuir la agresión al paciente y la estancia hospitalaria, y acelerar la recuperación.

Como todo nuevo procedimiento, exige una curva de aprendizaje. Esta comenzó con cursos en cadáveres para luego aplicar la técnica aprendida en pacientes seleccionados. Al analizar las complicaciones relacionadas con la experiencia del cirujano, detectamos que la mayor cantidad de complicaciones (12 casos, 24\%) se produjo en los primeros 16 pacientes, todas relacionadas con la vía de abordaje y no con la implantación de la caja, seguramente debido a la nueva forma de abordar la columna, diferente de los accesos tradicionales. Luego las complicaciones fueron disminuyendo exponencialmente hasta llegar al 6\%, pero estas últimas no estaban relacionadas con la vía de abordaje, sino más con errores en la técnica quirúrgica. 
En esta serie de 50 casos, la tasa de complicaciones operatorias tempranas en la cirugía mínimamente invasiva fue del $42 \%$, si la comparamos con el estudio de Rodgers y cols. ${ }^{2}$ en el que se analizaron las complicaciones tempranas de 600 casos de XLIF®, nuestros valores están muy por encima (42\% vs. 6,2\%), pero si tenemos en cuenta que solo el $8 \%$ de los pacientes que sufrieron complicaciones quedó con algún trastorno funcional crónico (complicaciones mayores) nos acercamos a su número. A su vez, Rodgers y cols. no tienen en cuenta a la debilidad del psoas como complicación, pues, para ellos, es algo habitual en el abordaje al incidir dicho músculo. Nosotros la consideramos una complicación, aunque menor, que aumentaría el porcentaje final.

En una serie de 84 casos, Youssef y cols. ${ }^{3}$ comunican un rango de complicaciones mayores del $4 \%$ al $8,6 \%$ y de complicaciones menores del $1,3 \%$ al 13,3\%, que coincide con nuestras tasas de complicaciones menores de entre el $2 \%$ y el $14 \%$, el dolor y la debilidad del muslo representan el mayor porcentaje ( 7 casos, $14 \%$ ).

La gran mayoría de los reportes sobre complicaciones de la vía lateral se refieren a los síntomas sensitivos, la debilidad y el dolor en el muslo, y comunican tasas que oscilan entre el 5\% y alrededor del 70\%, como el de Tohmeh y cols. ${ }^{4}$ quienes evaluaron a 102 pacientes sometidos a una fusión lateral con monitoreo neurofisiológico y detectaron un $17,6 \%$ de alguna alteración sensitiva posoperatoria en el muslo, con recuperación total dentro de los seis meses. O el estudio de Lee y cols. ${ }^{5}$ quienes evaluaron la fuerza de flexión de la cadera después de la fusión lumbar lateral en 33 pacientes, todos tuvieron alguna debilidad motora y una recuperación completa a las dos semanas de la cirugía. En nuestra estadística, los pacientes (7 casos, 14\%) mejoraron dentro del mes de la cirugía. Estos síntomas están muy relacionados con la apertura de las fibras del psoas o con la lesión directa en el abordaje de los nervios ilioinguinal o iliohipogástrico, que están situados en la pared abdominal y, a veces, del nervio genitofemoral, que se encuentra dentro del psoas. El monitoreo sensitivo de estos nervios es difícil y, para evitar o disminuir esta complicación, se debería tener cuidado en el manejo de la incisión y realizar una observación directa de los nervios durante el abordaje de la pared abdominal, si se presentan, separando, con suavidad, el retroperitoneo al entrar en la cavidad. En resumen, conocer muy bien la anatomía peritoneal y del plexo lumbar.

La lesión motora del plexo femoral está descrita como una de las complicaciones más devastadoras de este procedimiento. El nivel L4-L5 es considerado el más riesgoso, según varios autores, por la proximidad del nervio en el sitio ideal de colocación de la caja, tercio medio/posterior del disco. En el estudio retrospectivo de Cahill y cols., ${ }^{6}$ se determina la incidencia de lesión del nervio femoral en 118 pacientes y se comunica un 5\% de complicaciones con dicho nervio en el nivel L4-L5. Knight y cols. ${ }^{7}$ publicaron que dos de 58 pacientes con abordaje lateral quedaron con una secuela por lesión del nervio L4. Si bien nosotros no tuvimos ninguna complicación de este tipo, no podemos extraer muchas conclusiones, ya que solo accedimos a ese espacio en cuatro oportunidades, pero debemos tener en cuenta dos circunstancias muy importantes: la primera, la planificación y evaluación exhaustivas preoperatorias de las imágenes, mirando la resonancia magnética y la tomografía computarizada (sobre todo los cortes axiales y coronales) y la segunda, el tiempo de retracción del separador, no más de 20-30 min por nivel, como lo demostraron Bendersky y cols., ${ }^{8}$ ya que esto disminuye la neuropraxia del nervio por compresión de las valvas separadoras, que podría ocurrir por su proximidad a la discectomía.

Otra lesión compleja por la proximidad al cuerpo vertebral es la lesión vascular, se podría lesionar a los grandes vasos y arterias segmentarias. La mínima incisión interfiere con la reparación de un potencial daño vascular, relacionado con los grandes vasos, por su posición anterior, la cual podría ser fatal, como publicaron Assina y cols...$^{9}$ el primer reporte de muerte con lesión de la vena cava inferior por las valvas retractoras del separador.

En nuestra casuística, hubo solo un caso de lesión de una arteria segmentaria, que se pudo ligar, sin necesidad de abrir más la incisión. Santillan y cols.$^{10}$ reportaron un caso de lesión de la arteria segmentaria, con diagnóstico tardío, que requirió una embolización angiográfica posterior.

En estudios con mayores casuísticas, se comunican muy bajas incidencias de lesiones vasculares y, a su vez, la posición en decúbito lateral aleja los vasos del sitio operatorio lo que evitaría aún más un potencial daño. Esto lo ejemplifica muy bien el estudio de resonancia magnética de los movimientos de las estructuras abdominales en los cambios de posición supina a lateral, llevado a cabo por Deukmedjian y cols. ${ }^{11}$ Nosotros pensamos e insistimos en la importancia de evaluar las imágenes preoperatorias, esto nos ayuda a disminuir los riesgos y a valorar la viabilidad del procedimiento.

La subsidencia de las cajas es un fenómeno frecuente en la fusión intersomática lumbar, observado también en la vía lateral. La mayoría de los estudios se remiten a la calidad ósea de los pacientes, el tamaño de las cajas y la falta de fusión ósea, como factores relacionados.

Le y cols. ${ }^{12}$ reportaron una tasa de subsidencia radiográfica del $14,3 \%$ y clínica del $2,1 \%$ en 140 pacientes y concluyeron en que, con cajas de $22 \mathrm{~mm}$ y con fusiones posteriores con tornillos bilaterales, disminuirían estas 
complicaciones. Marchi y cols. ${ }^{13}$ sugieren colocar las cajas más anchas de 22 o $26 \mathrm{~mm}$ y no tan altas (8 o 10$)$ para evitar este riesgo. En nuestra investigación, colocamos todas cajas de $22 \mathrm{~mm}$ y tuvimos cuatro casos (8\%) de subsidencia: tres del platillo inferior y uno del superior, diagnosticados en el posoperatorio inmediato. Aunque todos fueron suplementados con una fijación posterior, igualmente se produjo el hundimiento de la caja hacia el cuerpo vertebral y esto lo atribuimos a un trabajo agresivo de los platillos vertebrales, cuando se prepara el espacio intersomático. Este sería otro factor que se agrega a los ya mencionados y para que no ocurra, hay que ser muy cuidadoso en la preparación del espacio intersomático y evitar la ruptura no intencional del platillo vertebral que, con mucha frecuencia, resulta afectado. Si bien podría haber más casos de subsidencia en controles alejados, este estudio evaluó solo las complicaciones tempranas.

Otra complicación infrecuente es la fractura del cuerpo vertebral. En el estudio de Brier-Jones y cols., ${ }^{14}$ se reportaron cuatro pacientes con fracturas intraoperatorias del cuerpo vertebral, todas por ruptura del platillo vertebral. Los autores lo atribuyen a la colocación de una placa lateral con tornillos muy cerca del platillo vertebral, esto llevaría a que se altere el hueso trabecular por debajo de este, debilitándolo y generando una sobrecarga que conduciría a la caja a fracturar el platillo. También otros autores asociaron la fractura del cuerpo a la colocación de una placa lateral de sostén, como muestran Kepler y cols. ${ }^{15}$ Nosotros tuvimos un solo caso, en el que no colocamos placa lateral, pero sí fijación pedicular posterior complementaria. Creemos que se debió a una violación del platillo inferior del disco superior al colocar la primera caja y luego a la debilitación del platillo superior del disco inferior al cuerpo de L3 al colocar la segunda caja, provocando el trazo de fractura longitudinal. Este tipo de complicaciones se podría evitar, como ya mencionamos, con el manejo cuidadoso en la preparación del platillo, procurando no debilitarlo. También al implantar las cajas intersomática se deberían utilizar las valvas protectoras de los platillos y no distraer excesivamente el espacio con implantes muy grandes, sobre todo en los pacientes con osteoporosis importante.

La migración lateral de la caja es una rara complicación del abordaje lateral. En la bibliografía, solo encontramos el reporte de un caso con una migración lateral de la caja, a pesar de la fijación posterior. ${ }^{16} \mathrm{El}$ cuadro era sintomático y fue necesaria una revisión quirúrgica para solucionarlo. Towers y Kurtom ${ }^{17}$ refieren un solo caso de migración lateral, pero sin fijación posterior y asintomática, que no requirió revisión. Esto último es similar a nuestro único caso de migración lateral en una fusión lateral L3-L4, pero con fijación posterior, el paciente no tenía síntomas y no hubo necesidad de una nueva cirugía.

Sabemos que, en esta técnica, uno realiza la resección de los ligamentos laterales, pero por la estabilidad propia de la caja al recuperar la altura del espacio, no debería migrar. Towers y Kurtom sospechan que la migración se puede deber a dos factores: la falta de una compresión posterior paralela con los tornillos pediculares al hacer la suplementación posterior o a la inadecuada resección del ligamento contralateral, lo que dejaría un mínimo desequilibrio coronal, con presión excesiva asimétrica sobre la caja, que favorece su salida. Daffner y Wang, y Towers y Kurtom sugieren colocar una placa lateral de sostén, sobre todo si hay gran desequilibrio coronal. Creemos que un buen trabajo del ligamento contralateral y un adecuado tamaño de la caja pueden lograr la estabilidad por sí misma, sin necesidad de colocar una placa lateral.

Como complicación muy poco frecuente, un paciente (2\%) tuvo una seudohernia (parálisis de los músculos de la pared abdominal) que mejoró espontáneamente dentro de los tres meses de la cirugía. Esto coincide con el estudio de Dakwar y cols. ${ }^{18}$ quienes comunicaron una incidencia del 1,8\% y ningún paciente con secuelas.

Tres nervios inervan los músculos de la pared abdominal (subcostal, iliohipogástrico e ilioinguinal), y se encuentran durante los primeros pasos del abordaje lateral (pared abdominal y grasa retroperitoneal). Sugerimos una divulsión roma y suave (evitar el electrobisturí) de los músculos del abdomen y de la separación del peritoneo, para evitar esta infrecuente complicación.

\section{CONCLUSIÓN}

Las mayores complicaciones ocurrieron en la vía de abordaje y fueron disminuyendo progresivamente, a medida que aumentaba la experiencia del cirujano y con el menor tiempo quirúrgico empleado, lo que torna más seguro y confiable a este tipo de procedimiento. 
Conflicto de intereses: Los autores no declaran conflictos de intereses.

ORCID de H. Resano: http:///rcid.org/0000-0002-2772-803X

\section{BIBLIOGRAFÍA}

1. Ozgur BM, Aryan HE, Pimenta L, Taylor WR. Extreme lateral interbody fusion (XLIF): a novel surgical technique for anterior lumbar interbody fusion. Spine J 2006;6(4):435-43. https://doi.org/10.1016/j.spinee.2005.08.012

2. Rodgers WB, Gerber EJ, Patterson J. Intraoperative and early postoperative complications in extreme lateral interbody fusion: an analysis of 600 cases. Spine (Phila PA 1976) 2011;36(1):26-32. https://doi.org/10.1097/ BRS.0b013e3181e1040a

3. Youssef JA, McAfee PC, Patty CA, Raley E, DeBauche S, Shucosky E, et al. Minimally invasive surgery: lateral approach interbody fusion: results and review. Spine (Phila PA 1976) 2010;35(26 Suppl):S302-11. https://doi. org/10.1097/BRS.0b013e3182023438

4. Tohmeh AG, Rodgers WB, Peterson MD. Dynamically evoked, discrete-threshold electromyography in the extreme lateral interbody fusion approach. J Neurosurg Spine 2011;14(1):31-7. https://doi.org/10.3171/2010.9.SPINE09871

5. Lee YP, Regev GJ, Chan J, Zhang B, Taylor W, Kim CW, et al. Evaluation of hip flexion strength following lateral lumbar interbody fusion. Spine J 2013;13(10):1259-62. https://doi.org/10.1016/j.spinee.2013.05.031

6. Cahill KS, Martinez JL, Wang MY, Vanni S, Levi AD. Motor nerve injuries following the minimally invasive lateral transpsoas approach. J Neurosurg Spine 2012;17(3):227-31. https://doi.org/10.3171/2012.5.SPINE1288

7. Knight RQ, Schwaegler P, Hanscom D, Roh J. Direct lateral lumbar interbody fusion for degenerative conditions: early complication profile. J Spinal Disord Tech 2009;22(1):34-7. https://doi.org/ https://doi.org/10.1097/ BSD.0b013e3181679b8a

8. Bendersky M, Solá C, Muntadas J, Gruenberg M, Calligaris S, Mereles M, et al. Monitoring lumbar plexus integrity in extreme lateral transpsoas approaches to the lumbar spine: a new protocol with anatomical bases. Eur Spine J 2015;24(5):1051-7. https://doi.org/10.1007/s00586-015-3801-9

9. Assina R, Majmundar NJ, Herschman Y, Heary RF. First report of major vascular injury due to lateral transpsoas approach leading to fatality. J Neurosurg Spine 2014;21(5):794-8. https://doi.org/10.3171/2014.7.SPINE131146

10. Santillan A, Patsalides A, Gobin YP. Endovascular embolization of iatrogenic lumbar artery pseudoaneurysm following extreme lateral interbody fusion (XLIF). Vasc Endovascular Surg 2010;44(7):601-3. https://doi. org/10.1177/1538574410374655

11. Deukmedjian AR, Le TV, Dakwar E, Martinez CR, Uribe JS. Movement of abdominal structures on magnetic resonance imaging during positioning changes related to lateral lumbar spine surgery: a morphometric study: clinical study. J Neurosurg Spine 2012;16(6):615-23. https://doi.org/10.3171/2012.3.SPINE1210

12. Le TV, Baaj AA, Dakwar E, Burkett CJ, Murray G, Smith DA, et al. Subsidence of polyetheretherketone intervertebral cages in mini- mally invasive lateral retroperitoneal transpsoas lumbar interbody fusion. Spine(Phila PA 1976) 2012;37(14):1268-73. https://doi.org/10.1097/BRS.0b013e3182458b2f

13. Marchi L, Abdala N, Oliveira L, Amaral R, Coutinho E, Pimenta L. Radiographic and clinical evaluation of cage subsidence after stand- alone lateral interbody fusion. J Neurosurg Spine 2013;19(1):110-8. https://doi. org/10.3171/2013.4.SPINE12319

14. Brier-Jones JE, Palmer DK, Ǐnceoğlu S, Cheng WK. Vertebral body fractures after transpsoas interbody fusion procedures. Spine J 2011;11(11):1068-72. https://doi.org/10.1016/j.spinee.2011.07.020

15. Kepler CK, Sharma AK, Huang RC. Lateral transpsoas interbody fusion (LTIF) with plate fixation and unilateral pedicle screws: a preliminary report. J Spinal Disord Tech 2011;24(6):363-7. https://doi.org/10.1097/ BSD.0b013e3181fee8b6

16. Daffner SD, Wang JC. Migrated XLIF cage: case report and discussion of surgical technique. Orthopedics 2010,33(7):518. https://doi.org/10.3928/01477447-20100526-21 
17. Towers WS, Kurtom KH. Stand-alone LLIF lateral cage migration: A case report. Cureus 2015;7(10):e347. https:// doi.org/10.7759/cureus.347

18. Dakwar E, Le TV, Baaj AA, Le AX, Smith WD, Akbarnia BA, et al. Abdominal wall paresis as a complication of minimally invasive lateral transpsoas interbody fusion. Neurosurg Focus 2011;31(4):E18. https://doi. org/10.3171/2011.7.FOCUS11164 